Hand/Peripheral Nerve Image

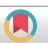

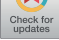

\section{Congenital bilateral second metacarpophalangeal joint stiffness}

\section{Sangho Oh}

Department of Plastic and Reconstructive Surgery, Keimyung University Dongsan Hospital, Daegu, Korea

\section{Correspondence: Sangho Oh}

Department of Plastic and Reconstructive Surgery, Keimyung University Dongsan Hospital, 1035 Dalgubeol-daero, Dalseo-gu, Daegu 42601, Korea Tel: +82-53-258-4581, Fax: +82-53-258-4583

E-mail: fwmesser@hanmail.net

Received: May 22, 2020 • Revised: December 1, 2020 •

Accepted: December 2, 2020

pISSN: 2234-6163・ elSSN: 2234-6171

https://doi.org/10.5999/aps.2020.00906• Arch Plast Surg 2021:48:293-294

Copyright (C) 2021 The Korean Society of Plastic and Reconstructive Surgeons This is an Open Access article distributed under the terms of the Creative Commons Attribution Non-Commercial License (https://creativecommons.org/licenses/by-nc/4.0/ which permits unrestricted non-commercial use, distribution, and reproduction in any medium, provided the original work is properly cited.

Hand movement, especially in the fingers, is very important for quality of life [1]. In the fingers, the metacarpophalangeal joint (MCPJ) and proximal interphalangeal joint are more important functionally than the distal interphalangeal joint [2]. In this article, we report a rare case of a patient with congenital bilateral stiffness in the second MCPJ, inherited from the mother, that needed no surgery or intervention. This is the first reported case of congenital bilateral second MCPJ stiffness without any external trauma history.

A 30-year-old man visited the hospital because of finger stiffness. No visual abnormalities of his hand were noted, but the patient could not fully flex his second MCPJ actively (Table 1, Fig. 1).

Fig. 2 illustrates extrinsic tightness in both index fingers. The right side was more severely impacted

Table 1. Range of motion of the second metacarpophalangeal joint in the mother and son

\begin{tabular}{llcc}
\hline \multirow{2}{*}{ Case } & & \multicolumn{2}{c}{ Range of motion $\left(^{\circ}\right)$} \\
\cline { 3 - 4 } & & Active & Passive \\
\hline \multirow{2}{*}{ Son } & Right & $0-10$ & $0-30$ \\
& Left & $0-20$ & $0-45$ \\
\multirow{2}{*}{ Mother } & Right & $0-40$ & $0-65$ \\
& Left & $0-45$ & $0-65$
\end{tabular}
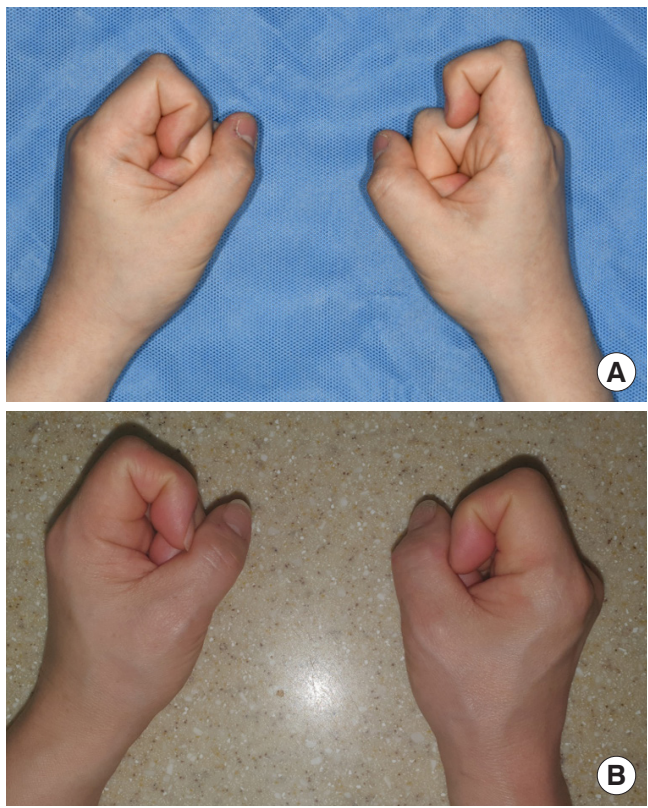

Fig. 1.

Images of both hands making a fist for the son $(\mathrm{A})$ and mother (B). Both index fingers protruded more than the other fingers when making a fist on both sides.
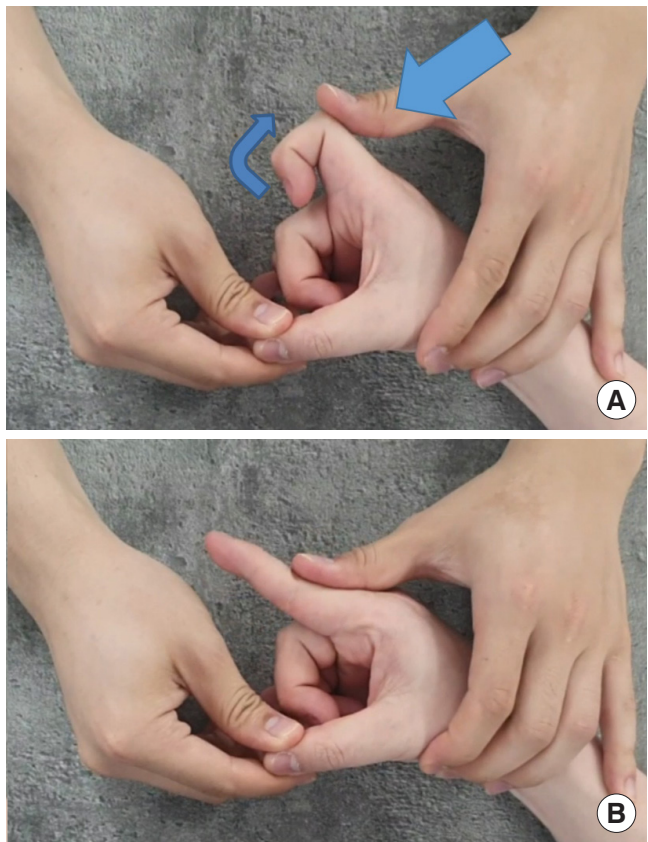

Fig. 2.

(A, B) Extrinsic tightness test on the right second metacarpophalangeal joint (MCPJ). After the examiner pushed the patient's index finger to flex the MCPJ in the proximal interphalangeal joint (PIPJ) and distal interphalangeal joint (DIPJ) flexed positions, the MCPJ could not be flexed without full extension of the PIPJ and DIPJ. than the left side.

On an X-ray examination, ultrasonography, and magnetic resonance imaging, there were no abnormalities except for a bump on both second metacarpal heads on the X-ray examination and right MCPJ dorsal capsule contracture on ultrasonography. The mother's X-rays also showed bumps on both second metacarpal heads (Fig. 3).

Because of the long duration of the stiffness, the patient had to adjust his finger use, substituting his middle finger for his index finger in all motions due to stiffness in the second MCPJ. To increase the range of 

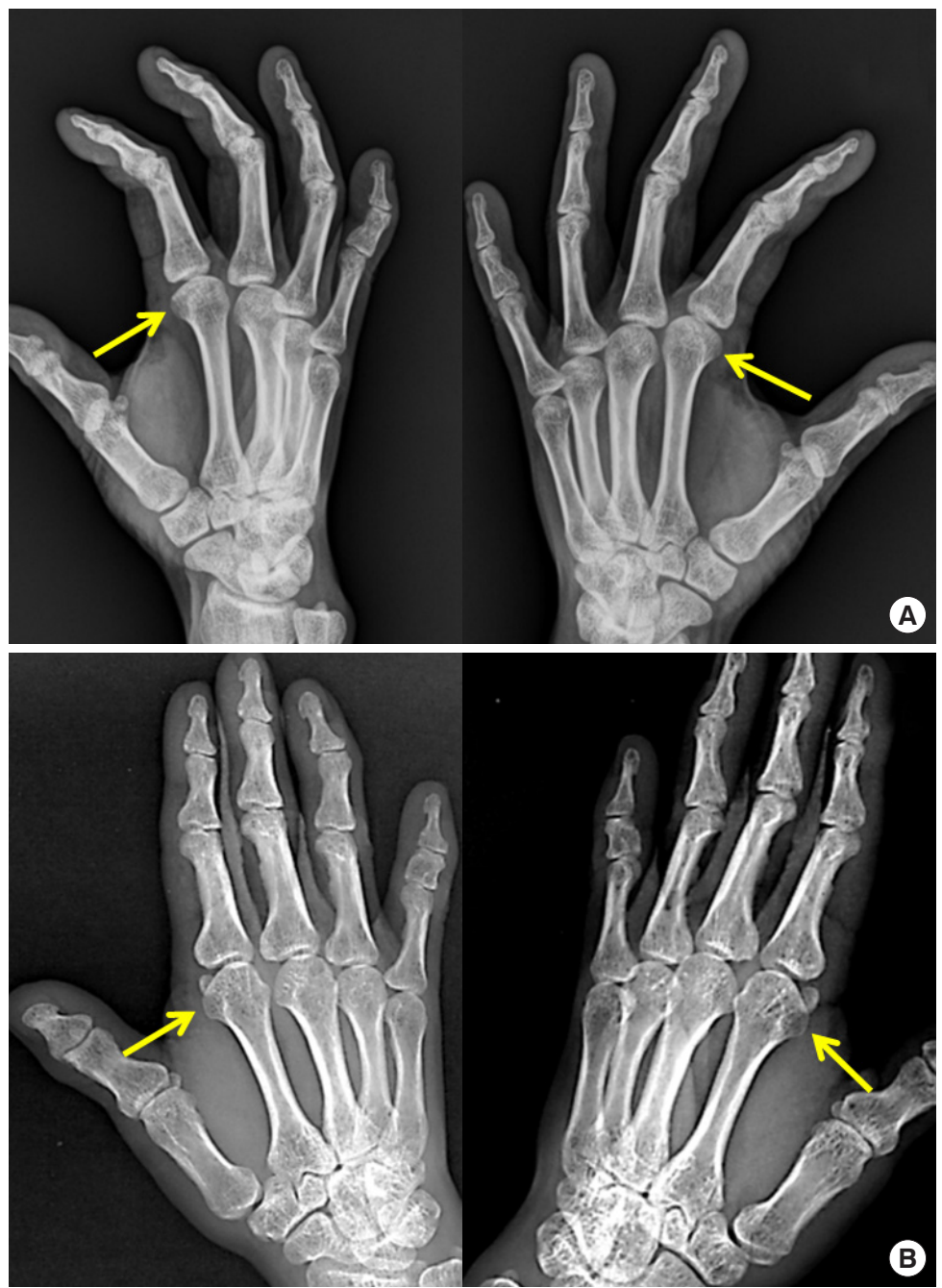

Fig. 3.

$\mathrm{X}$-ray views (anteroposterior and oblique) of both hands for the son $(A)$ and mother $(B)$. It was noted that there were volar bumps of both second metacarpal heads. The yellow arrow shows the bony bump on the metacarpal heads.

motion of the second MCPJ, corrective surgery may be needed. However, in some situations, surgery can worsen a patient's functional status. In our opinion, the choice of whether to perform surgery in such cases should be made based on the patient's condition.

\section{Notes}

\section{Conflict of interest}

No potential conflict of interest relevant to this article was reported.

\section{Ethical approval}

The study was approved by the Institutional Review Board of Keimyung University (IRB No. DSMC 2020-09-070) and performed in accordance with the principles of the Declaration of Helsinki. Written informed consent was obtained.

\section{Patient consent}

The patients provided written informed consent for the publication and the use of their images.

\section{ORCID}

Sangho Oh https://orcid.org/0000-0002-8739-908X

\section{References}

1. Yang G, McGlinn EP, Chung KC. Management of the stiff finger: evidence and outcomes. Clin Plast Surg 2014;41:501-12.

2. Carlsen BT, Bakri K, Al-Mufarrej FM, et al.

Osteoarthritis in the hand and wrist. In: Peter CN, David HS, editors. Plastic surgery. London: Elsevier Saunders; 2013. p. 411-48. 